\title{
Free Vibration and Hardening Behavior of Truss Core Sandwich Beam
}

\author{
J. E. Chen, ${ }^{1}$ W. Zhang, ${ }^{2}$ M. Sun, ${ }^{3}$ and M. H. Yao ${ }^{2}$ \\ ${ }^{1}$ Tianjin Key Laboratory of the Design and Intelligent Control of the Advanced Mechatronical System, \\ Tianjin University of Technology, Tianjin 300384, China \\ ${ }^{2}$ College of Mechanical Engineering, Beijing University of Technology, Beijing 100124, China \\ ${ }^{3}$ School of Science, Tianjin Chengjian University, Tianjin 300384, China \\ Correspondence should be addressed to M. Sun; sunmin0537@163.com
}

Received 1 October 2015; Revised 19 February 2016; Accepted 6 March 2016

Academic Editor: Emiliano Mucchi

Copyright (C) 2016 J. E. Chen et al. This is an open access article distributed under the Creative Commons Attribution License, which permits unrestricted use, distribution, and reproduction in any medium, provided the original work is properly cited.

\begin{abstract}
The dynamic characteristics of simply supported pyramidal truss core sandwich beam are investigated. The nonlinear governing equation of motion for the beam is obtained by using a Zig-Zag theory. The averaged equations of the beam with primary, subharmonic, and superharmonic resonances are derived by using the method of multiple scales and then the corresponding frequency response equations are obtained. The influences of strut radius and core height on the linear natural frequencies and hardening behaviors of the beam are studied. It is illustrated that the first-order natural frequency decreases continuously and the second-order and third-order natural frequencies initially increase and then decrease with the increase of strut radius, and the first three natural frequencies all increase with the rise of the core height. Furthermore, the results indicate that the hardening behaviors of the beam become weaker with the increase of the rise of strut radius and core height. The mechanisms of variations in hardening behavior of the sandwich beam with the three types of resonances are detailed and discussed.
\end{abstract}

\section{Introduction}

Truss core sandwich structures are drawing more and more attention from researchers due to their excellent mechanical properties and many functional applications. Cellular materials have always played a significant role in many engineering fields. As a new member of the family of cellular materials, truss-like material has broad application prospects. Evans et al. [1] compared the integrated performances of the different types of cellular materials; the advantages of mechanical characteristics and multifunction of the lattice materials were highlighted. Deshpande and Fleck [2] investigated the collapse responses of the sandwich beams with truss cores. They revealed that truss core sandwich beams were significantly lighter than the sandwich beams with metallic foam core against the design constraint of collapse load. Wallach and Gibson [3] analyzed the elastic moduli, uniaxial compressive strengths, and shear strength of three-dimensional truss structures. It was concluded that the truss material had improved properties over the closed-cell aluminum foam.
Next, the manufacturing techniques, mechanical behaviors, and functionalities of the sandwich structures with different truss cores were paid close attention to by researchers from different research fields [4-9].

Unfortunately, the investigations on vibration responses of the truss core sandwich structures are extremely limited. Lou et al. [10] studied the free vibration of the simply supported truss core sandwich beams; the natural frequencies of the sandwich beams were obtained and the theoretical results agree well with the numerical results. Xu and Qiu [11] investigated the free vibration of the truss core sandwich beams with interval parameters. The effects of geometric and material parameters on the natural frequencies were analyzed. Lou et al. [12] analyzed the effects of local damage on vibration characteristics of the truss core sandwich structures by numerical and experimental methods. Li and Lyu [13] investigated the active vibration control of lattice sandwich beams with pyramidal lattice core using the piezoelectric actuator/sensor pairs. Song and Li [14] studied the nonlinear aeroelastic characteristics and the active flutter control of 
sandwich beams. The face sheets and piezoelectric material were modeled by the Euler-Bernoulli beam theory, and the core was modeled by the Reddy third-order shear deformation theory.

Moreover, the vibration behaviors of the truss core sandwich structures can be deduced with the help of a large number of dynamical studies on the sandwich structures with honeycomb core, foam core, and other cores. Frostig and Thomsen [15] used high-order sandwich panel theory to analyze the free vibration of sandwich panels with a flexible core. Biglari and Jafari [16] studied the free vibrations of doubly curved sandwich panels based on a refined threelayered theory. Jam et al. [17] investigated the free vibration of sandwich panels by using an improved high-order theory. In several papers like the aforementioned three references, the three-dimensional elasticity theory was used for the core, which has a better accuracy to predict the response of the thick sandwich structures.

For thin and some moderately thick sandwich structures, the equivalent single layer theory and the layerwise theory were widely used. E. Nilsson and A. C. Nilsson [18] investigated the dynamic properties of sandwich structures with honeycomb and foam cores. Li and Zhu [19] studied the free vibration of honeycomb sandwich plates by using the improved Reddy third-order shear deformation plate theory. J. H. Zhang and W. Zhang [20] investigated the global bifurcations and multipulse chaotic dynamics of a simply supported honeycomb sandwich rectangular plate by using the extended Melnikov method. Yang et al. [21] investigated the transverse vibrations and stability of an axially moving sandwich beam. Sahoo and Singh [22] analyzed the buckling and free vibration analysis of the laminated composite and sandwich plates by using a new trigonometric Zig-Zag theory. Khare et al. [23] presented the free vibration analysis of composite and sandwich laminates by using a high-order shear deformation theory. Sakiyama et al. [24] studied the free vibration of a three-layer sandwich beam with an elastic or viscoelastic core and arbitrary boundary conditions. Banerjee [25] analyzed the free vibration of three-layered symmetric sandwich beams by using the dynamic stiffness method. Ćetković and Vuksanović [26] studied the bending, free vibrations, and buckling of the laminated composite and sandwich plates by using the generalized layerwise theory of Reddy. Ferreira [27] analyzed the mechanical behaviors of the laminated composite and sandwich plates by using a layerwise shear deformation theory and the multiquadrics discretization method.

Although the studies on the vibrations of the sandwich structures are relatively detailed, the unique dynamic characteristics, especially the nonlinear behaviors of the sandwich structures with truss core, are still required to be explored. The main aim of this paper is to particularly study the linear and nonlinear dynamic behaviors of the truss core sandwich beam. In this paper, the nonlinear governing equation of motion for the simply supported truss core sandwich beam with immovable edges is obtained by using the von Karman type equation, Hamilton's principle, and a Zig-Zag theory. The influences of strut radius and core height on the linear

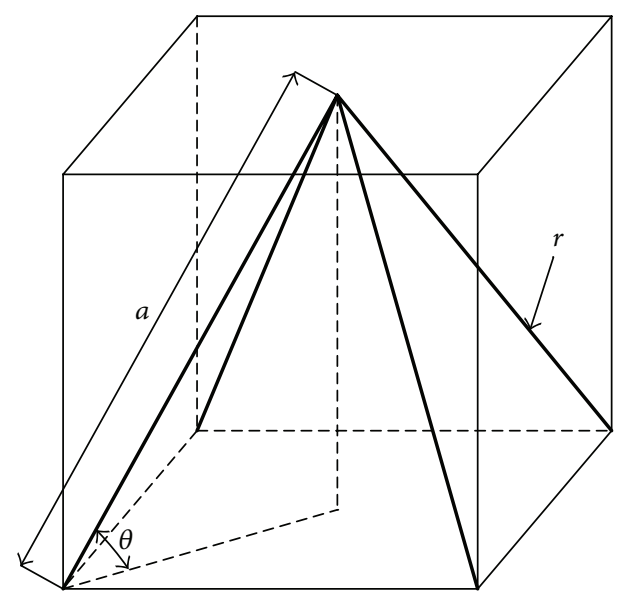

FIGURE 1: Sketches of a unit cell of the sandwich beam with pyramidal truss core.

natural frequencies and hardening behaviors of the sandwich beam are investigated. The nonlinear frequency responses of the sandwich beam with primary, subharmonic, and superharmonic resonances are obtained and the numerical results are discussed.

\section{Formulation}

The simply supported sandwich beam with pyramidal truss core subjected to a transverse uniform load is considered in the paper. A unit cell of the pyramidal truss core is shown in Figure 1 . The length, radius, and inclination angle of the truss are represented by $a, r$, and $\theta$, respectively. Thus, the relative density $\bar{\rho}$ and the equivalent shear modulus $G$ are expressed as follows [2]:

$$
\begin{aligned}
& \bar{\rho}=\frac{\rho_{c}}{\rho}=\frac{2 \pi}{\cos ^{2} \theta \sin \theta}\left(\frac{r}{a}\right)^{2}, \\
& G=\pi \sin \theta\left(\frac{r}{a}\right)^{2} E,
\end{aligned}
$$

where $\rho_{c}$ is the equivalent density of the truss core and $\rho$ and $E$ are the density and elasticity modulus of the mother material of the truss.

The truss core sandwich beam can be modeled as a laminated beam which is composed of two solid layers and an equivalent continuum core layer. Generally, for a sandwich structure, the following assumptions [28] can be given:

(1) The thickness of the truss core sandwich beam remains constant during deformation.

(2) Only bending deformation is considered for both thin face sheets and only shear deformation is considered for the core.

(3) The face sheets and truss core are combined closely. Thus, the deflections between each layer are continuous.

These assumptions are widely used to study the sandwich structures which have thin face sheets and thick core. Based 


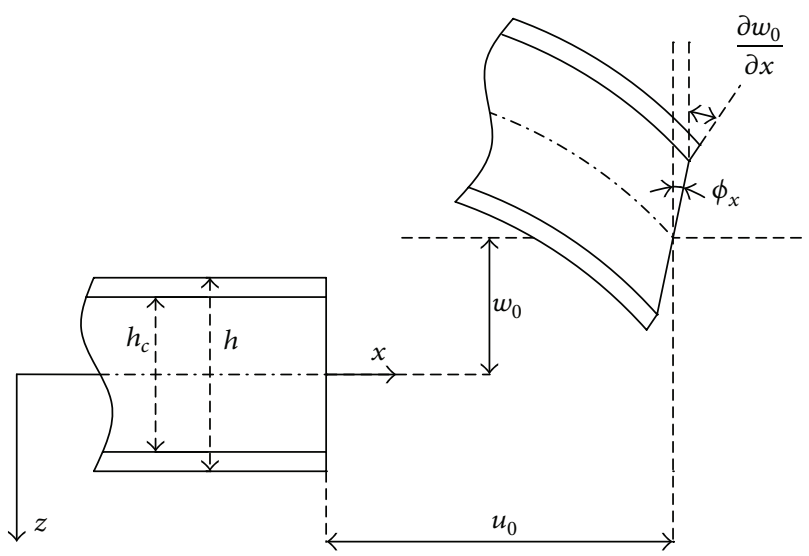

FIGURE 2: Undeformed and deformed geometries of the truss core sandwich beam.

on undeformed and deformed geometries of the truss core sandwich beam as shown in Figure 2, the displacement field can be expressed in terms of the midplane displacements $u_{0}$ and $w_{0}$ and rotations $\phi_{x}$, the face sheets of the sandwich beam adopt the Kirchhoff hypothesis, and the truss core adopts the first-order shear deformation theory. Hence,

$$
\begin{aligned}
-\frac{h}{2} \leq z & \leq-\frac{h_{c}}{2}, \\
u_{t} & =u_{0}-z \frac{\partial w_{0}}{\partial x}+\frac{h_{c}}{2}\left(\phi_{x}-\frac{\partial w_{0}}{\partial x}\right), \\
w_{t} & =w_{0}, \\
-\frac{h_{c}}{2} \leq z & \leq \frac{h_{c}}{2}, \quad u_{c}=u_{0}-z \phi_{x}, \\
\frac{h_{c}}{2} \leq z & \leq \frac{h}{2}, \quad w_{c}=w_{0}, \\
u_{b} & =u_{0}-z \frac{\partial w_{0}}{\partial x}-\frac{h_{c}}{2}\left(\phi_{x}-\frac{\partial w_{0}}{\partial x}\right), \\
w_{b} & =w_{0}, \quad
\end{aligned}
$$

where the subscripts $t, c$, and $b$ represent the top face sheet, the truss core, and the bottom face sheet, respectively. $h$ represents the total thickness of the truss core sandwich beam and $h_{c}$ represents the height of the truss core.

Using von Karman's theory for the geometric nonlinearity, the strain-displacement relations have the form

$$
\begin{aligned}
& \varepsilon_{x x 1}=\frac{\partial u_{t}}{\partial x}+\frac{1}{2}\left(\frac{\partial w_{t}}{\partial x}\right)^{2}, \\
& \gamma_{x z}=\frac{\partial u_{c}}{\partial z}+\frac{\partial w_{c}}{\partial x}, \\
& \varepsilon_{x x 3}=\frac{\partial u_{b}}{\partial x}+\frac{1}{2}\left(\frac{\partial w_{b}}{\partial x}\right)^{2},
\end{aligned}
$$

where $\varepsilon_{x x 1}$ and $\varepsilon_{x x 3}$ represent the strains of the face sheets and $\gamma_{x z}$ represents the strain of the truss core.

Substituting (2a), (2b), and (2c) into (3), we have

$$
\begin{aligned}
& \left\{\begin{array}{l}
\varepsilon_{x x 1} \\
\varepsilon_{x x 3} \\
\gamma_{x z}
\end{array}\right\}=\left\{\begin{array}{c}
\varepsilon_{x x 1}^{(0)} \\
\varepsilon_{x x 3}^{(0)} \\
\gamma_{x z}^{(0)}
\end{array}\right\}+z\left\{\begin{array}{c}
\varepsilon_{x x 1}^{(1)} \\
\varepsilon_{x x 3}^{(1)} \\
\gamma_{x z}^{(1)}
\end{array}\right\}, \\
& \left\{\begin{array}{c}
\varepsilon_{x x 1}^{(0)} \\
\varepsilon_{x x 3}^{(0)} \\
\gamma_{x z}^{(0)}
\end{array}\right\} \\
& =\left\{\begin{array}{l}
\frac{\partial u_{0}}{\partial x}+\frac{h_{c}}{2}\left(\frac{\partial \phi_{x}}{\partial x}-\frac{\partial^{2} w_{0}}{\partial x^{2}}\right)+\frac{1}{2}\left(\frac{\partial w_{0}}{\partial x}\right)^{2} \\
\frac{h_{c}}{2}\left(\frac{\partial \phi_{x}}{\partial x}-\frac{\partial^{2} w_{0}}{\partial x^{2}}\right)+\frac{1}{2}\left(\frac{\partial w_{0}}{\partial x}\right)^{2}
\end{array}\right\}, \\
& \left\{\begin{array}{l}
\varepsilon_{x x 1}^{(1)} \\
\varepsilon_{x x 3}^{(1)} \\
\gamma_{x z}^{(1)}
\end{array}\right\}=\left\{\begin{array}{c}
\partial w_{0} \\
-\frac{\partial^{2} w_{0}}{\partial x^{2}} \\
-\frac{\partial^{2} w_{0}}{\partial x^{2}} \\
0
\end{array}\right\} .
\end{aligned}
$$

The mathematical statement of Hamilton's principle is given as

$$
\int_{t_{1}}^{t_{2}}(\delta U+\delta W-\delta K) d t=0 .
$$

The virtual kinetic energy $\delta K$, virtual strain energy $\delta U$, and the virtual work $\delta W$ are, respectively, given by

$$
\begin{aligned}
\delta U & =\delta U_{1}+\delta U_{2}+\delta U_{3}, \\
\delta K & =\delta K_{1}+\delta K_{2}+\delta K_{3}, \\
\delta W & =-\int_{A} F \delta w_{0}-\mu \dot{w}_{0} \delta w_{0} d x d y \\
\delta U & =\int_{0}^{l} \int_{-h / 2}^{-h_{c} / 2} \sigma_{x x 1} \delta \varepsilon_{x x 1} d x d z \\
& +\int_{0}^{l} \int_{-h_{c} / 2}^{h_{c} / 2} \sigma_{x z} \delta \gamma_{x z} d x d z+\int_{0}^{l} \int_{h_{c} / 2}^{h / 2} \sigma_{x x 3} \delta \varepsilon_{x x 3} d x d z \\
& =\int_{0}^{l} \int_{-h / 2}^{-h_{c} / 2} \sigma_{x x 1}\left(\delta \varepsilon_{x x 1}^{(0)}+z \delta \varepsilon_{x x 1}^{(1)}\right) d x d z \\
& +\int_{0}^{l} \int_{-h_{c} / 2}^{h_{c} / 2} \sigma_{x z} \delta \gamma_{x z} d x d z+\int_{0}^{l} \int_{h_{c} / 2}^{h / 2} \sigma_{x x 3}\left(\delta \varepsilon_{x x 3}^{(0)}\right. \\
& \left.+z \delta \varepsilon_{x x 3}^{(1)}\right) d x d z=\int_{0}^{l} N_{x x 1} \delta \varepsilon_{x x 1}^{(0)} \\
& +M_{x x 1} \delta \varepsilon_{x x 1}^{(1)} d x+\int_{0}^{l} \int_{-h_{c} / 2}^{h_{c} / 2} Q_{x} \delta \gamma_{x z} d x+\int_{0}^{l} N_{x x 3} \\
& +\delta \varepsilon_{x x 3}^{(0)}+M_{x x 3} \delta \varepsilon_{x x 3}^{(1)} d x d z,
\end{aligned}
$$




$$
\begin{aligned}
\delta K & =\int_{0}^{l} \int_{-h / 2}^{-h_{c} / 2} \rho\left[\left(\dot{u}_{0}-z \frac{\partial \dot{w}_{0}}{\partial x}+\frac{h_{c}}{2} \dot{\phi}_{x}-\frac{h_{c}}{2} \frac{\partial \dot{w}_{0}}{\partial x}\right)\right. \\
& \cdot\left(\delta \dot{u}_{0}-z \frac{\partial \delta \dot{w}_{0}}{\partial x}+\frac{h_{c}}{2} \delta \dot{\phi}_{x}-\frac{h_{c}}{2} \frac{\partial \delta \dot{w}_{0}}{\partial x}\right) \\
& \left.+\dot{w}_{0} \delta \dot{w}_{0}\right] d x d z \\
& +\int_{0}^{l} \int_{-h_{c} / 2}^{h_{c} / 2} \rho_{c}\left[\left(\dot{u}_{0}-z \dot{\phi}_{x}\right)\left(\delta \dot{u}_{0}-z \delta \dot{\phi}_{x}\right)\right. \\
& \left.+\dot{w}_{0} \delta \dot{w}_{0}\right] d x d z \\
& +\int_{0}^{l} \int_{h_{c} / 2}^{h / 2} \rho\left[\left(\dot{u}_{0}-z \frac{\partial \dot{w}_{0}}{\partial x}-\frac{h_{c}}{2} \dot{\phi}_{x}+\frac{h_{c}}{2} \frac{\partial \dot{w}_{0}}{\partial x}\right)\right. \\
& +\left(\delta \dot{u}_{0}-z \frac{\partial \delta \dot{w}_{0}}{\partial x}-\frac{h_{c}}{2} \delta \dot{\phi}_{x}+\frac{h_{c}}{2} \frac{\partial \delta \dot{w}_{0}}{\partial x}\right) \\
& \left.+\dot{w}_{0} \delta \dot{w}_{0}\right] d x d z,
\end{aligned}
$$

where the superposed dot on a variable indicates its first derivative with respect to time: $\dot{u}_{0}=\partial u_{0} / \partial t$, and so on.

Substituting (6a), (6b), and (6c) into (5), the nonlinear governing equations of motion for the truss core sandwich beam are obtained:

$$
\begin{aligned}
& \left(N_{x x 1}+N_{x x 3}\right)_{, x}=\left(2 I_{01}+I_{02}\right) \ddot{u}_{0}-I_{12} \ddot{\phi}_{x}, \\
& \frac{h_{c}}{2}\left(N_{x x 1}-N_{x x 3}\right)_{, x}+Q_{x}=\left(\frac{h_{c}^{2}}{2} I_{01}+I_{22}\right) \ddot{\phi}_{x} \\
& \quad-\left(\frac{h_{c}^{2}}{2} I_{01}+h_{c} I_{11}\right) \frac{\partial \ddot{w}_{0}}{\partial x}-I_{12} \ddot{u}_{0}, \\
& \left(N_{x x 1} \frac{\partial w_{0}}{\partial x}+N_{x x 3} \frac{\partial w_{0}}{\partial x}\right)_{, x}+\frac{h_{c}}{2}\left(N_{x x 1}-N_{x x 3}\right)_{, x x} \\
& \quad+\left(M_{x x 1}+M_{x x 3}\right)_{, x x}+Q_{x, x}+F \\
& \quad-\mu \dot{w}_{0}=\left(\frac{h_{c}^{2}}{2} I_{01}+h_{c} I_{11}\right) \frac{\partial \ddot{\phi}_{x}}{\partial x} \\
& \quad-\left(\frac{h_{c}^{2}}{2} I_{01}+2 h_{c} I_{11}+2 I_{21}\right) \frac{\partial^{2} \ddot{w}_{0}}{\partial x^{2}} \\
& \quad+\left(2 I_{01}+I_{02}\right) \ddot{w}_{0},
\end{aligned}
$$

where

$$
\begin{aligned}
& \left\{\begin{array}{l}
I_{01} \\
I_{11} \\
I_{21}
\end{array}\right\}=\int_{-h / 2}^{-h_{c} / 2}\left\{\begin{array}{c}
1 \\
z \\
z^{2}
\end{array}\right\} \rho d z, \\
& \left\{\begin{array}{l}
I_{02} \\
I_{12} \\
I_{22}
\end{array}\right\}=\int_{-h_{c} / 2}^{h_{c} / 2}\left\{\begin{array}{c}
1 \\
z \\
z^{2}
\end{array}\right\} \rho_{c} d z,
\end{aligned}
$$

$$
\begin{aligned}
N_{x x 1} & =\int_{-h / 2}^{-h_{c} / 2} \sigma_{x x 1} d z, \\
N_{x x 3} & =\int_{h_{c} / 2}^{h / 2} \sigma_{x x 3} d z, \\
Q_{x} & =\int_{-h_{c} / 2}^{h_{c} / 2} \sigma_{x z} d z, \\
M_{x x 1} & =\int_{-h / 2}^{-h_{c} / 2} z \sigma_{x x 1} d z, \\
M_{x x 3} & =\int_{h_{c} / 2}^{h / 2} z \sigma_{x x 3} d z .
\end{aligned}
$$

We consider the face sheets and the truss core are made of the same isotropy material. The stress-strain relations of the truss core sandwich beam can be represented by

$$
\begin{gathered}
\sigma_{x x 1}=E \varepsilon_{x x 1}, \\
\sigma_{x x 3}=E \varepsilon_{x x 3}, \\
\sigma_{x z}=G \gamma_{x z} .
\end{gathered}
$$

The stress-displacement relations can be expressed as

$$
\begin{aligned}
N_{x x 1} & =A_{1} \varepsilon_{x x 1}^{(0)}+B_{1} \varepsilon_{x x 1}^{(1)}, \\
N_{x x 3} & =A_{3} \varepsilon_{x x 3}^{(0)}+B_{3} \varepsilon_{x x 3}^{(1)}, \\
M_{x x 1} & =B_{1} \varepsilon_{x x 1}^{(0)}+D_{1} \varepsilon_{x x 1}^{(1)}, \\
M_{x x 3} & =B_{3} \varepsilon_{x x 3}^{(0)}+D_{3} \varepsilon_{x x 3}^{(1)}, \\
Q_{x} & =C \gamma_{x z}^{(0)},
\end{aligned}
$$

where

$$
\begin{aligned}
\left(A_{1}, B_{1}, D_{1}\right) & =\int_{-h / 2}^{-h_{c} / 2} E\left(1, z, z^{2}\right) d z, \\
\left(A_{3}, B_{3}, D_{3}\right) & =\int_{h_{c} / 2}^{h / 2} E\left(1, z, z^{2}\right) d z, \\
C & =\int_{-h_{c} / 2}^{h_{c} / 2} G d z .
\end{aligned}
$$

$A_{1}$ and $A_{3}$ are called extensional stiffness, $B_{1}$ and $B_{3}$ are called bending-extensional stiffness, $D_{1}$ and $D_{3}$ are called bending stiffness, $C$ is called shear stiffness, and $I_{i j}$ are the mass moments of inertia.

Considering the symmetry of the two face sheets of the truss core sandwich beam about the $x$-axis, we have

$$
\begin{aligned}
& A_{1}=A_{3}, \\
& B_{1}=-B_{3}, \\
& D_{1}=D_{3} .
\end{aligned}
$$


Using the stress-displacement relations, (7a)-(7c) can be expressed in terms of generalized displacements:

$$
\begin{aligned}
& 2 A_{1} \frac{\partial^{2} u_{0}}{\partial x^{2}}+2 A_{1} \frac{\partial w_{0}}{\partial x} \frac{\partial^{2} w_{0}}{\partial x^{2}}=\left(2 I_{01}+I_{02}\right) \ddot{u}_{0}-I_{12} \ddot{\phi}_{x} \\
& \frac{h_{c}^{2}}{2} A_{1} \frac{\partial^{2} \phi_{x}}{\partial x^{2}}-\frac{h_{c}^{2}}{2} A_{1} \frac{\partial^{3} w_{0}}{\partial x^{3}}-h_{c} B_{1} \frac{\partial^{3} w_{0}}{\partial x^{3}}-C \phi_{x} \\
& \quad+C \frac{\partial w_{0}}{\partial x}=\left(\frac{h_{c}^{2}}{2} I_{01}+I_{22}\right) \ddot{\phi}_{x} \\
& \quad-\left(\frac{h_{c}^{2}}{2} I_{01}+h_{c} I_{11}\right) \frac{\partial \ddot{w}_{0}}{\partial x}-I_{12} \ddot{u}_{0} \\
& \left.+\frac{h_{c}^{2}}{2} A_{1}+B_{1} h_{c}\right) \frac{\partial^{3} \phi_{x}}{\partial x^{3}} \\
& \quad+\left(-\frac{h_{c}^{2}}{2} A_{1}-2 B_{1} h_{c}-2 D_{1}\right) \frac{\partial^{4} w_{0}}{\partial x^{4}}-C \frac{\partial \phi_{x}}{\partial x} \\
& \quad+\left(\frac{h_{c}^{2}}{2} I_{01}+2 h_{c} I_{11}+2 I_{21}\right) \frac{\partial^{2} \ddot{w}_{0}}{\partial x^{2}} \\
& \quad+2 A_{01}+3 \dot{w}_{0}=\left(\frac{\partial u_{0}}{\partial x} \frac{\partial^{2} w_{0}}{\partial x^{2}}+F\right. \\
& \left.\quad+\frac{\partial w_{0}}{\partial x}\right)_{01}^{2} \frac{\partial^{2} w_{0}}{\partial x^{2}}+2 A_{1} \frac{\partial^{2} u_{0}}{\partial x^{2}} \frac{\partial w_{0}}{\partial x}
\end{aligned}
$$

\section{Validation}

In order to check the accuracy of the presented model, the natural frequencies are calculated and compared with the results presented by [10]. For linear vibration, the in-plane motion of the structure can be neglected. Therefore, the simply supported boundary conditions of the sandwich beam can be expressed as

$$
w_{0}=\frac{\partial \phi_{x}}{\partial x}=M_{x x 1}=M_{x x 3}=0 \quad \text { at } x=0, l .
$$

The displacements $\phi_{x}$ and $w_{0}$ which satisfy (13) can be represented as

$$
\begin{aligned}
& \phi_{x}=\sum_{n=1}^{N} \phi_{n}(t) \cos \frac{n \pi x}{l}, \\
& w_{0}=\sum_{n=1}^{N} w_{n}(t) \sin \frac{n \pi x}{l} .
\end{aligned}
$$

We only consider the transverse vibration of the truss core sandwich beam; thus, we can neglect the rotatory inertia terms. Substituting (14a) and (14b) into the linear form of
TABLE 1: Comparison of natural frequencies of the sandwich beam with the first group of parameters.

\begin{tabular}{lcccc}
\hline Mode & Ahmed & Hwu & Lou & Present \\
\hline 1 & 56 & 58 & 57 & 57.14 \\
2 & - & 221 & 219 & 219.58 \\
3 & 451 & 459 & 465 & 465.17 \\
4 & - & 742 & 767 & 768.18 \\
5 & 1073 & 1048 & 1105 & 1106.69 \\
\hline
\end{tabular}

TABLE 2: Comparison of natural frequencies of the sandwich beam with the second group of parameters.

\begin{tabular}{lcc}
\hline Mode & Lou & Present \\
\hline 1 & 137.74 & 137.81 \\
2 & 523.09 & 523.95 \\
3 & 1091.38 & 1094.11 \\
\hline
\end{tabular}

(12a), (12b), and (12c) and applying the Galerkin method, the natural frequencies are obtained, as shown in Tables 1 and 2. The good agreement between the present and published results validates the accuracy of the present method.

The dimensions and material properties in Table 1 are

$$
\begin{aligned}
E_{f} & =68.9 \mathrm{GPa}, \\
h_{f} & =0.4572 \mathrm{~mm}, \\
\rho_{f} & =2680 \mathrm{~kg} / \mathrm{m}^{3}, \\
G_{c} & =0.08268 \mathrm{GPa}, \\
h_{c} & =12.7 \mathrm{~mm}, \\
\rho_{c} & =32.8 \mathrm{~kg} / \mathrm{m}^{3}, \\
l & =0.9144 \mathrm{~m} .
\end{aligned}
$$

The dimensions and material properties in Table 2 are

$$
\begin{aligned}
E_{f} & =210 \mathrm{GPa}, \\
h_{f} & =1 \mathrm{~mm}, \\
\rho_{f} & =7930 \mathrm{~kg} / \mathrm{m}^{3}, \\
G_{c} & =1.03667 \mathrm{GPa}, \\
h_{c} & =15 \mathrm{~mm}, \\
\rho_{c} & =313.1739 \mathrm{~kg} / \mathrm{m}^{3}, \\
l & =0.6364 \mathrm{~m} .
\end{aligned}
$$

\section{Nonlinear Frequency Response Equation}

In the following analysis, the nonlinear vibrations of the truss core sandwich beam with simply supported boundary conditions are considered. For nonlinear vibration, the simply 
supported boundary conditions with immoveable edges of the beam can be expressed as

$$
u_{0}=w_{0}=\frac{\partial \phi_{x}}{\partial x}=M_{x x 1}=M_{x x 3}=0 \quad \text { at } x=0, l .
$$

The transformations of variables and parameters are introduced to obtain the dimensionless equation:

$$
\begin{aligned}
& \bar{u}_{0}=\frac{u_{0}}{l}, \\
& \bar{w}_{0}=\frac{w_{0}}{h}, \\
& \bar{\phi}_{x}=\phi_{x} \\
& \bar{x}=\frac{x}{l}, \\
& \bar{F}=\frac{l^{4}}{E h^{4}} F, \\
& \bar{\mu}=\frac{l}{h}\left(\frac{1}{\rho E}\right)^{1 / 2} \mu \text {, } \\
& \bar{t}=\frac{h}{l^{2}}\left(\frac{E}{\rho}\right)^{1 / 2} t, \\
& \bar{\Omega}=\frac{l^{2}}{h}\left(\frac{\rho}{E}\right)^{1 / 2} \Omega, \\
& \bar{h}_{c}=\frac{h_{c}}{h}, \\
& \bar{A}_{i}=\frac{l}{E h^{2}} A_{i} \text {, } \\
& \bar{B}_{i}=\frac{l}{E h^{3}} B_{i}, \\
& \bar{C}=\frac{l}{E h^{2}} C, \\
& \bar{D}_{i}=\frac{l}{E h^{4}} D_{i}, \\
& \bar{I}_{t i}=\frac{1}{l^{(t+1)} \rho} I_{t i}, \quad t=0,1,2 .
\end{aligned}
$$

Therefore, the dimensionless equations of motion for the truss core sandwich beam are obtained. For convenience of our study, we drop the overbar in the following analysis. The displacements $u_{0}, \phi_{x}$, and $w_{0}$ which satisfy (17) can be represented as

$$
\begin{aligned}
& u_{0}=\sum_{n=1}^{N} u(t) \sin (2 n \pi x), \\
& \phi_{x}=\sum_{n=1}^{N} \phi(t) \cos (n \pi x), \\
& w_{0}=\sum_{n=1}^{N} w(t) \sin (n \pi x) .
\end{aligned}
$$

In addition, the excitation has the form

$$
F=\sum_{n=1}^{N} f(t) \sin (n \pi x) .
$$

Next, the Galerkin method is used to obtain the nonlinear ordinary differential equations of motion for the sandwich beam. The transverse vibrations of the sandwich beam are mainly considered. In the same way, we can neglect the longitude and rotatory inertia terms. Substituting the displacements $u_{0}, \phi_{x}$, and $w_{0}$ into the resulting equations, the ordinary differential equation is derived:

$$
\begin{aligned}
\ddot{w}_{n} & +\mu_{n} \dot{w}_{n}+\omega_{n}^{2} w_{n}+\alpha_{n} w_{n}^{3}+g_{n}\left(w_{1}, w_{2}, \ldots, w_{N}\right) \\
& =\beta_{n} f_{n} \cos \left(\Omega_{n} t\right), \quad n=1,2, \ldots, N,
\end{aligned}
$$

where $g_{n}\left(w_{1}, w_{2}, \ldots, w_{N}\right)$ represents the coupled relation between the different modes and the coupled term does not appear when the single mode response is considered. The coefficients in (20) are presented in the Appendix.

The following scales transformations must be introduced for obtaining a system which is suitable for the application of the method of multiple scales and studying the resonances of the sandwich beam:

$$
\begin{aligned}
& \mu_{n} \longrightarrow \varepsilon \mu_{n}, \\
& \alpha_{n} \longrightarrow \varepsilon \alpha_{n}, \\
& \beta_{n} \longrightarrow \varepsilon \beta_{n},
\end{aligned}
$$

where $\varepsilon$ is a small, dimensionless parameter.

Because the coupled term $g_{n}\left(w_{1}, w_{2}, \ldots, w_{N}\right)$ has no contribution to the primary resonance of each mode in theoretical analysis, therefore, we can neglect this term and obtain the following equation:

$$
\ddot{w}_{n}+\varepsilon \mu_{n} \dot{w}_{n}+\omega_{n}^{2} w_{n}+\varepsilon \alpha_{n} w_{n}^{3}=\varepsilon \beta_{n} f_{n} \cos \left(\Omega_{n} t\right) .
$$

The method of multiple scales [29] is used to obtain the uniform solution of (22) in the following form:

$$
w_{n}(t, \varepsilon)=x_{n 0}\left(T_{0}, T_{1}\right)+\varepsilon x_{n 1}\left(T_{0}, T_{1}\right)+\cdots,
$$

where $T_{0}=t$ and $T_{1}=\varepsilon t$.

Then, we have the differential operators

$$
\begin{aligned}
\frac{d}{d t} & =\frac{\partial}{\partial T_{0}} \frac{\partial T_{0}}{\partial t}+\frac{\partial}{\partial T_{1}} \frac{\partial T_{1}}{\partial t}+\cdots=D_{0}+\varepsilon D_{1}+\cdots, \\
\frac{d^{2}}{d t^{2}} & =\left(D_{0}+\varepsilon D_{1}+\cdots\right)^{2}=D_{0}^{2}+2 \varepsilon D_{0} D_{1}+\cdots,
\end{aligned}
$$

where $D_{0}=\partial / \partial T_{0}$ and $D_{1}=\partial / \partial T_{1}$.

We have the following relation:

$$
\Omega_{n}=\omega_{n}+\varepsilon \sigma_{n} .
$$

$\sigma_{n}$ is the detuning parameter which quantitatively describes the nearness of excitation frequency to the linear natural frequency.

Substituting (23)-(25) into (22) and balancing the coefficients of the like power of $\varepsilon$ yield the following differential equations: 
$\varepsilon^{0}$ order:

$$
D_{0}^{2} x_{n 0}+\omega_{n}^{2} x_{n 0}=0 .
$$

$\varepsilon^{1}$ order:

$$
\begin{aligned}
D_{0}^{2} x_{n 1}+\omega_{n}^{2} x_{n 1}= & -2 D_{0} D_{1} x_{n 0}-\mu_{n} D_{0} x_{n 0}-\alpha_{n} x_{n 0}^{3} \\
& +\beta_{n} f_{n} \cos \left(\omega_{n} T_{0}+\sigma_{n} T_{1}\right) .
\end{aligned}
$$

The solution of (26a) in the complex form can be expressed as

$$
x_{n 0}=A_{n}\left(T_{1}\right) e^{i \omega_{n} T_{0}}+\overline{A_{n}}\left(T_{1}\right) e^{-i \omega_{n} T_{0}},
$$

where $\overline{A_{n}}$ is the complex conjugate of $A_{n}$.

Substituting (27) into (26b) yields

$$
\begin{aligned}
& D_{0}^{2} x_{n 1}+\omega_{n}^{2} x_{n 1}=\left(-2 i \omega_{n} D_{1} A_{n}-\mu_{n} i \omega_{n} A_{n}\right. \\
& \left.-3 \alpha_{n} A_{n}^{2} \overline{A_{n}}+\frac{\beta_{n} f_{n}}{2} e^{i \sigma_{n} T_{1}}\right) e^{i \omega_{n} T_{0}}+\mathrm{cc}+\mathrm{NST},
\end{aligned}
$$

where symbol cc denotes the parts of the complex conjugate of function on the right-hand side of (28) and NST represents the terms that do not produce secular terms.

Function $A_{n}$ may be denoted in the complex form:

$$
A_{n}=\frac{1}{2} a_{n} e^{i \varphi_{n}} .
$$

Substituting (29) into simplified (28) and separating the real and imaginary parts from the resulting equations, the two-dimensional averaged equation in the polar form is obtained as

$$
\begin{array}{r}
\dot{a}_{n}=-\frac{\mu_{n} a_{n}}{2}+\frac{\beta_{n} f_{n} \sin \left(\sigma_{n} T_{1}-\varphi_{n}\right)}{2 \omega_{n}}, \\
a_{n} \dot{\varphi}_{n}=\frac{3 \alpha_{n} a_{n}^{3}}{8 \omega_{n}}-\frac{\beta_{n} f_{n} \cos \left(\sigma_{n} T_{1}-\varphi_{n}\right)}{2 \omega_{n}} .
\end{array}
$$

Letting

$$
\gamma_{n}=\sigma_{n} T_{1}-\varphi_{n}
$$

we have

$$
\begin{gathered}
\dot{a}_{n}=-\frac{\mu_{n} a_{n}}{2}+\frac{\beta_{n} f_{n} \sin \gamma_{n}}{2 \omega_{n}}, \\
a_{n} \dot{\gamma}_{n}=a_{n} \sigma_{n}-\frac{3 \alpha_{n} a_{n}^{3}}{8 \omega_{n}}+\frac{\beta_{n} f_{n} \cos \gamma_{n}}{2 \omega_{n}} .
\end{gathered}
$$

Steady-state motions occur when $\dot{a}_{n}$ and $\dot{\gamma}_{n}$ equal zero, which correspond to the singular points of averaged (32a) and (32b). Thus, $a_{n}$ and $\gamma_{n}$ are constants. Eliminating $\gamma_{n}$ by using the relations between trigonometric functions, we obtain the frequency response equation of the truss core sandwich beam with primary resonance as follows:

$$
\left(\frac{\mu_{n} a_{n}}{2}\right)^{2}+\left(\sigma_{n} a_{n}-\frac{3 \alpha_{n} a_{n}^{3}}{8 \omega_{n}}\right)^{2}=\left(\frac{\beta_{n} f_{n}}{2 \omega_{n}}\right)^{2} .
$$

Subsequently, the subharmonic resonance and the superharmonic resonance of the sandwich beams are considered. In the two cases, the following two transformations are required:

$$
\begin{aligned}
& \mu_{n} \longrightarrow \varepsilon \mu_{n}, \\
& \alpha_{n} \longrightarrow \varepsilon \alpha_{n} .
\end{aligned}
$$

For subharmonic resonance, the detuning parameter $\sigma_{n}$ is introduced according to

$$
\Omega_{n}=3 \omega_{n}+\varepsilon \sigma_{n} .
$$

Similarly, the averaged equations in the case are obtained:

$$
\begin{aligned}
\dot{a}_{n}= & -\frac{\mu_{n} a_{n}}{2}-\frac{3 \alpha_{n} \beta_{n} a_{n}^{2} \Lambda \sin \gamma_{n}}{4 \omega_{n}}, \\
a_{n} \dot{\gamma}_{n}= & a_{n}\left(\sigma_{n}-\frac{9 \alpha_{n} \beta_{n}^{2} \Lambda^{2}}{\omega_{n}}\right)-\frac{9 \alpha_{n} a_{n}^{3}}{8 \omega_{n}} \\
& -\frac{9 \alpha_{n} \beta_{n} \Lambda a_{n}^{2} \cos \gamma_{n}}{4 \omega_{n}},
\end{aligned}
$$

where $\Lambda=(1 / 2) f_{n}\left(\omega_{n}^{2}-\Omega_{n}^{2}\right)^{-1}$.

The frequency response equation of the truss core sandwich beam with subharmonic resonance is

$$
\begin{aligned}
& \left(-\frac{\mu_{n} a_{n}}{2}\right)^{2}+\frac{1}{9}\left[a_{n}\left(\sigma_{n}-\frac{9 \alpha_{n} \beta_{n}^{2} \Lambda^{2}}{\omega_{n}}\right)-\frac{9 \alpha_{n} a_{n}^{3}}{8 \omega_{n}}\right]^{2} \\
& =\frac{9 \alpha_{n}^{2} \beta_{n}^{2} a_{n}^{4} \Lambda^{2}}{16 \omega_{n}^{2}} .
\end{aligned}
$$

For superharmonic resonance, the detuning parameter $\sigma_{n}$ is introduced according to

$$
3 \Omega_{n}=\omega_{n}+\varepsilon \sigma_{n} .
$$

The averaged equations in the case are obtained:

$$
\begin{aligned}
\dot{a}_{n}= & -\frac{\mu_{n} a_{n}}{2}-\frac{\alpha_{n} \beta_{n}^{3} \Lambda^{3} \sin \gamma_{n}}{\omega_{n}}, \\
a_{n} \dot{\gamma}_{n}= & a_{n}\left(\sigma_{n}-\frac{3 \alpha_{n} \beta_{n}^{2} \Lambda^{2}}{\omega_{n}}\right)-\frac{3 \alpha_{n} a_{n}^{3}}{8 \omega_{n}} \\
& -\frac{\alpha_{n} \beta_{n}^{3} \Lambda^{3} \cos \gamma_{n}}{\omega_{n}} .
\end{aligned}
$$

The frequency response equation of the truss core sandwich beam with superharmonic resonance is

$$
\begin{aligned}
& \left(-\frac{\mu_{n} a_{n}}{2}\right)^{2}+\left[a_{n}\left(\sigma_{n}-\frac{3 \alpha_{n} \beta_{n}^{2} \Lambda^{2}}{\omega_{n}}\right)-\frac{3 \alpha_{n} a_{n}^{3}}{8 \omega_{n}}\right]^{2} \\
& =\frac{\alpha_{n}^{2} \beta_{n}^{6} \Lambda^{6}}{\omega_{n}^{2}} .
\end{aligned}
$$

The plot of $a_{n}$ as a function of $\sigma_{n}$ for given $f$ is called a nonlinear frequency response curve which can be used to describe the hardening behavior of a structure. In the following analysis, the parameters in (33), (37), and (40) should be calculated by using the Appendix. 


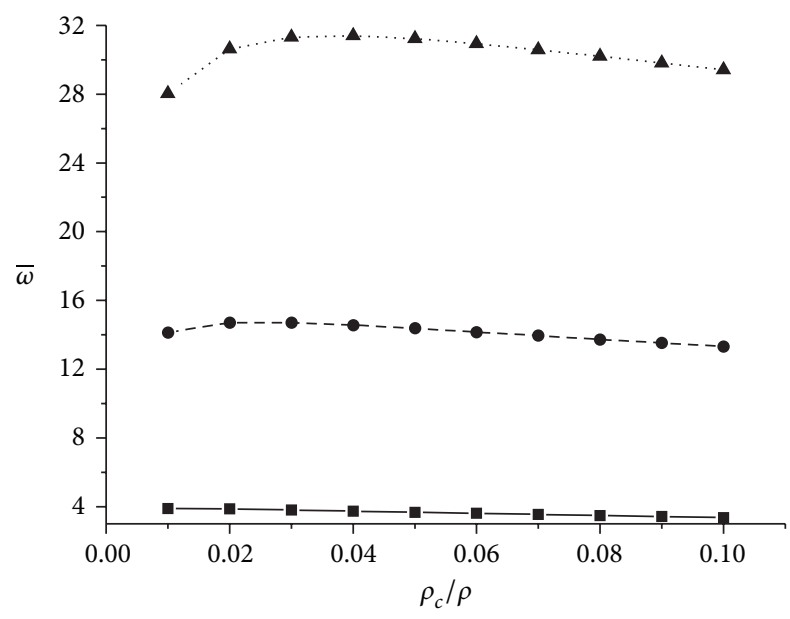

FIGURE 3: Variations of dimensionless linear natural frequencies with respect to the strut radius. Solid line: first-order frequency; dash line: second-order frequency; dot-dash line: third-order frequency.

\section{Numerical Results}

In the study, the influences of the structural parameters on the dimensionless linear natural frequencies of the truss core sandwich beam are studied at first by neglecting the nonlinear terms in (20). The face sheets and the truss of the sandwich beam are made of the same material. The material properties are taken as follows: $E=68.9 \mathrm{GPa}$ and $\rho=$ $2680 \mathrm{~kg} / \mathrm{m}^{3}$. Figure 3 indicates that the first-order natural frequency decreases continuously and the second-order and third-order natural frequencies initially increase and then decrease with the increase of strut radius. The relative density corresponding to the turning point in the changing curve of the second-order natural frequency is smaller than that of the third-order natural frequency. When the strut radius increases, the mass moment of inertia and shear stiffness of the sandwich beam both increase; the changes of the natural frequencies depend upon the combined effect of the two factors. The structural parameters in the figure are given as $h=0.012 \mathrm{~m}, h_{c}=0.01 \mathrm{~m}, \theta=\pi / 4$, and $l=0.7 \mathrm{~m}$.

Figure 4 shows that the first three natural frequencies all increase with the rise of the core height and the higherorder natural frequency increases faster than the lower-order natural frequency. When the core height increases, the mass moment of inertia, extensional stiffness, bending stiffness, and shear stiffness of the sandwich beam all decrease; the changes of the natural frequencies depend upon the combined effect of those factors. The structural parameters in the figure are given as $h=0.012 \mathrm{~m}, r=0.6 \mathrm{~mm}, \theta=\pi / 4$, and $l=0.7 \mathrm{~m}$.

The steady-state responses of the first three modes of the sandwich beam with primary resonance are investigated. The hardening-type nonlinearity of the first three modes all becomes weaker with increasing strut radius and core height, as shown in Figures 5 and 6 . The bending of the frequency response curve mainly depends on the nonlinear term and the frequency parameter according to (33). It can be known

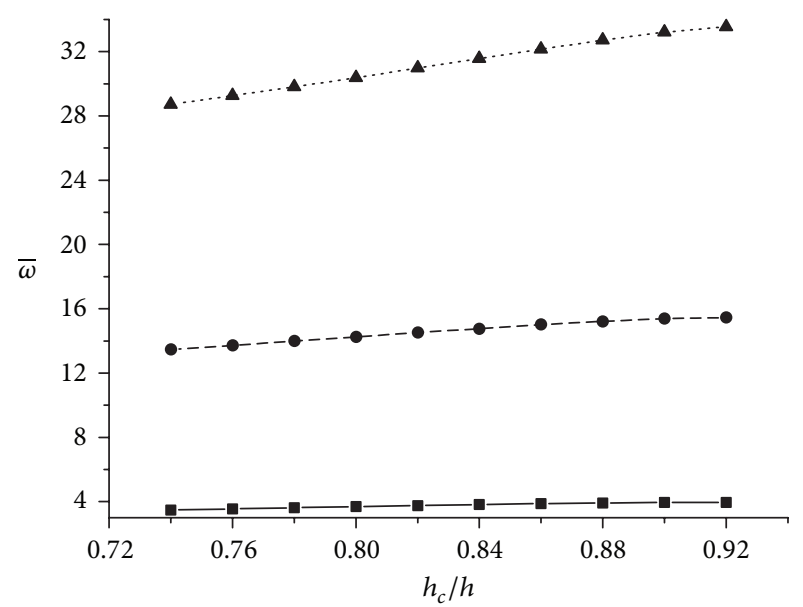

FIGURE 4: Variations of dimensionless linear natural frequencies with respect to the core height. Solid line: first-order frequency; dash line: second-order frequency; dot-dash line: third-order frequency.

from $\alpha_{n}$ in the Appendix that the nonlinear term is related to the extensional stiffness of the face sheets as well as the mass moment of inertia, thickness, and length of the sandwich beam. The theoretical analyses indicate that the reasons for the weakening of the hardening-type nonlinearity with the rise of strut radius and core height are different.

At first, we discuss the influence of strut radius on the hardening behavior of the sandwich beam. With the increase of the strut radius, the hardening behavior of the beam is weakened by the rise of the mass moment of inertia and shear stiffness. It is concluded from (33) that the increases of the mass moment of inertia and shear stiffness both weaken the hardening behavior. The structural parameters in Figure 5 are the same as in Figure 3. With the increase of the core height, the hardening behavior of the beam is weakened. It is concluded that the decrease of extensional stiffness tends to weaken the hardening behavior and the decrease of the mass moment of inertia, shear stiffness, and bending stiffness tends to strengthen the hardening behavior of the sandwich beam. Obviously, the effect of the extensional stiffness is dominant according to Figure 6 . The structural parameters in this figure are the same as in Figure 4.

Furthermore, the hardening-type nonlinearity of the higher mode is stronger than that of the lower mode, and the influences of the structural parameters on the hardening behavior for the lower mode are slightly larger than for the higher mode. In the analysis of the primary resonance of the sandwich beam, $\mu=0.001$ and $f=1$ are always chosen.

Subharmonic and superharmonic resonances are relatively easy to be generated at the lower modes, especially at the first mode. The nonlinear frequency response curves for the first mode of the sandwich beam with subharmonic and superharmonic resonances are investigated in the paper; the numerical results are shown in Figures 7 and 8, respectively. Figure 7 demonstrates that the subharmonic responses only occur in the frequency ranges which are higher than the linear natural frequency no matter which and how structural parameters change. Increasing strut radius and core height 


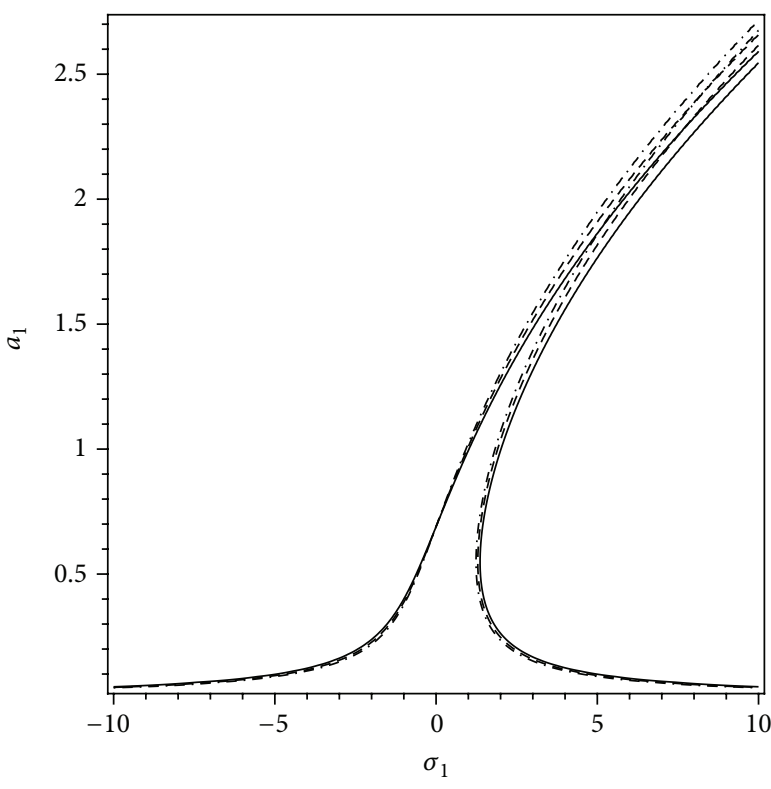

(a)

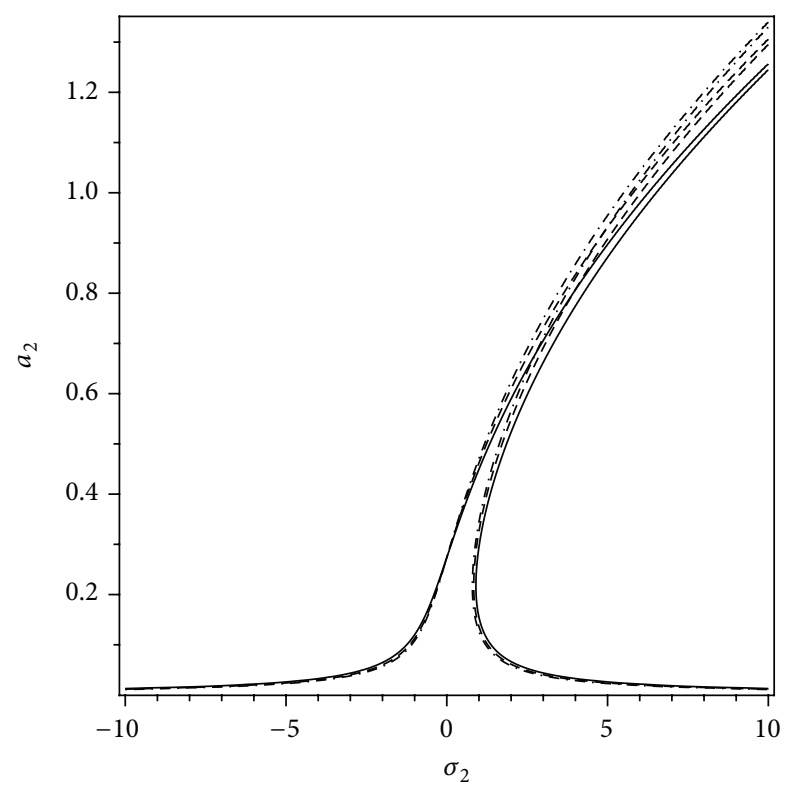

(b)

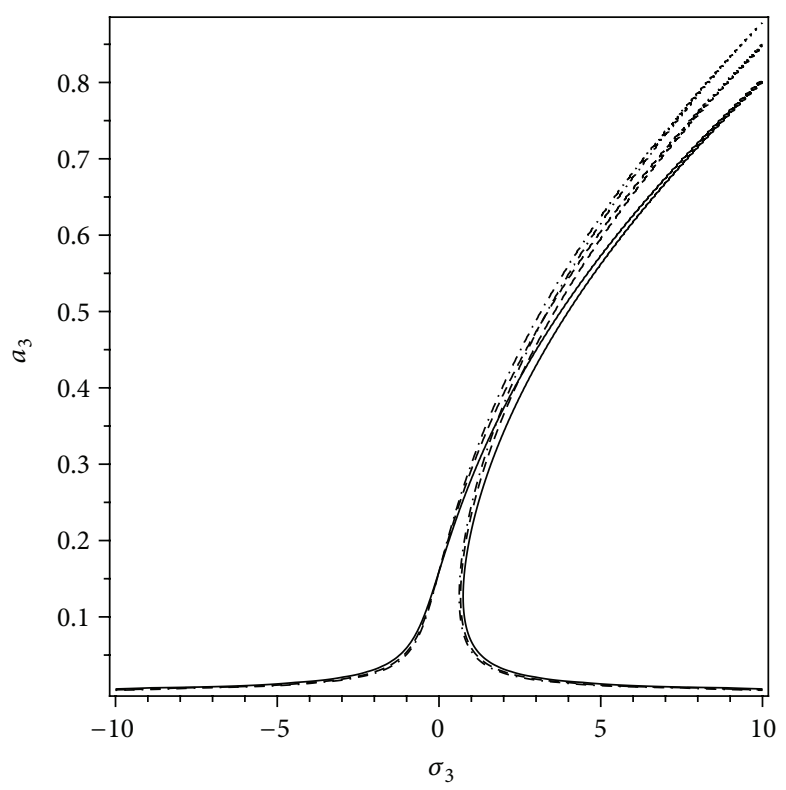

(c)

FiguRE 5: Influences of the strut radius on the hardening behavior of the first three modes: (a) the first mode; (b) the second mode; (c) the third mode. Solid line: $\bar{\rho}=0.02$; dash line: $\bar{\rho}=0.04$; dot-dash line: $\bar{\rho}=0.06$.

will weaken the hardening-type nonlinearity in the resonance case, just as the influences of the structural parameters on the responses of the beam with primary resonance. Figure 8 demonstrates that the shapes of the frequency response curves for the first mode with superharmonic responses are similar to that of the sandwich beam with primary resonance. The variations in the structural parameters can not only affect the hardening behaviors of the beam but also significantly change the frequency range of the resonance responses.
Decreasing strut radius and core height can broaden the frequency ranges of the resonance responses.

Based on the analysis of the frequency response equations, we conclude that, in the cases of subharmonic and superharmonic resonances, the mechanism of the variation of the hardening behavior via the structural parameters is the same as that in the case of primary resonance. $\mu=0.001$ and $f=2$ are chosen in Figures 7 and 8. Moreover, the structural parameters in Figures 7(a) and 8(a) are the same 


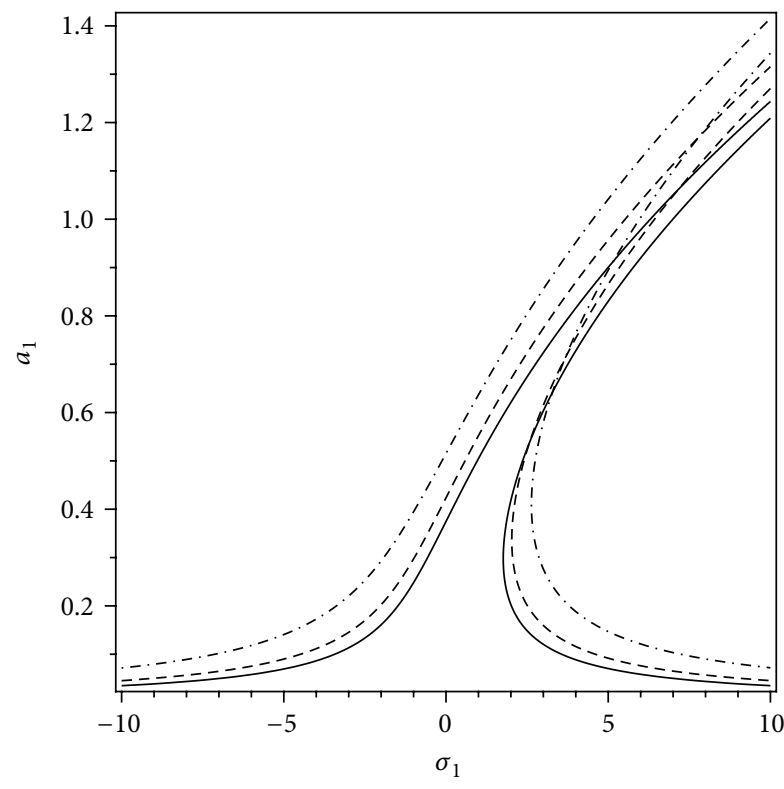

(a)

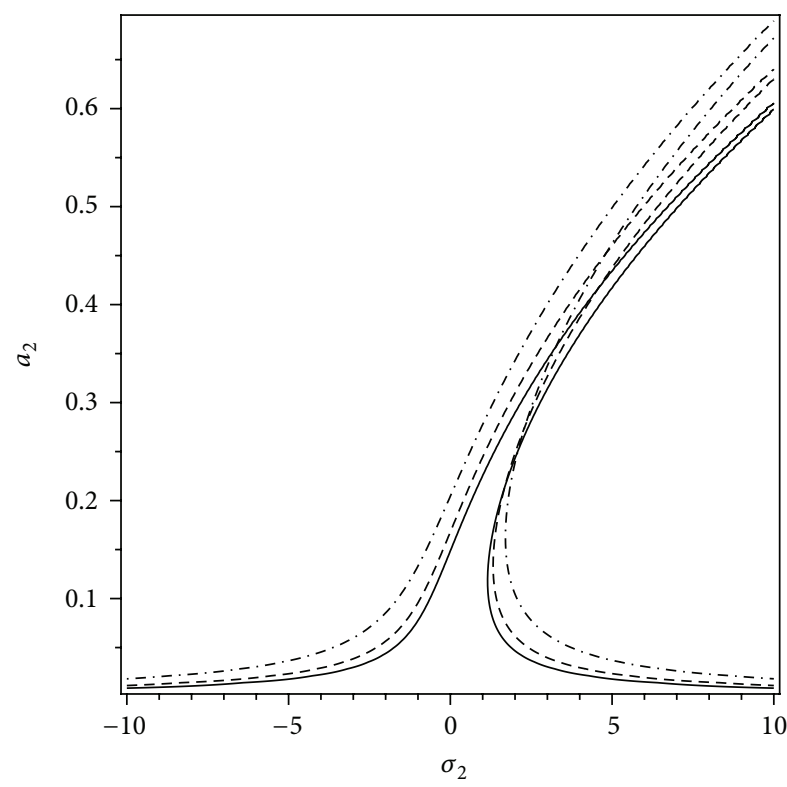

(b)

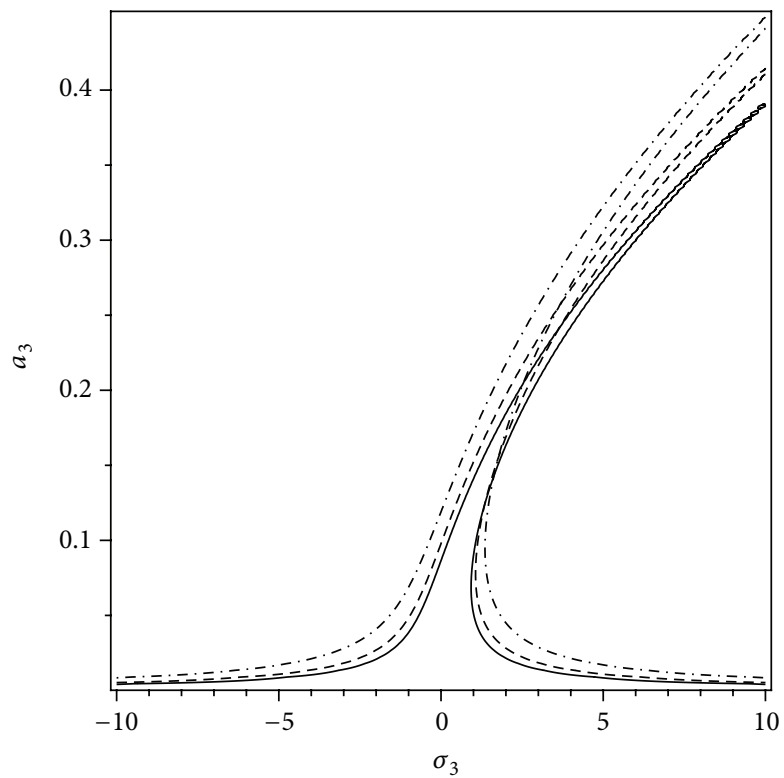

(c)

Figure 6: Influences of the core height on the hardening behavior of the first three modes: (a) the first mode; (b) the second mode; (c) the third mode. Solid line: $h_{c} / h=0.74$; dash line: $h_{c} / h=0.82$; dot-dash line: $h_{c} / h=0.9$.

as in Figure 3, and the structural parameters in Figures 7 (b) and $8(\mathrm{~b})$ are the same as in Figure 4.

\section{Conclusions}

The natural frequency and hardening behavior of sandwich beam with pyramidal truss core are investigated. The truss core is equivalent to a continuous homogeneous material and a Zig-Zag theory is used to derive the nonlinear governing equation of motion for the sandwich beam. At first, the natural frequencies are calculated to validate the present model, and the changing rules of the natural frequency with the strut radius and core height are obtained. It is illustrated that the first-order natural frequency decreases continuously and the second-order and third-order natural frequencies initially increase and then decrease with the increase of strut radius, and the first three natural frequencies all increase with the rise of the core height. Then, the hardening behaviors of the sandwich beam are analyzed. Numerical simulations indicate that the effects of the two structural parameters on the hardening behavior in the cases of primary resonance, subharmonic resonance, and superharmonic resonance are similar. However, the mechanisms of the variations of the hardening behavior are very different. The influences of 


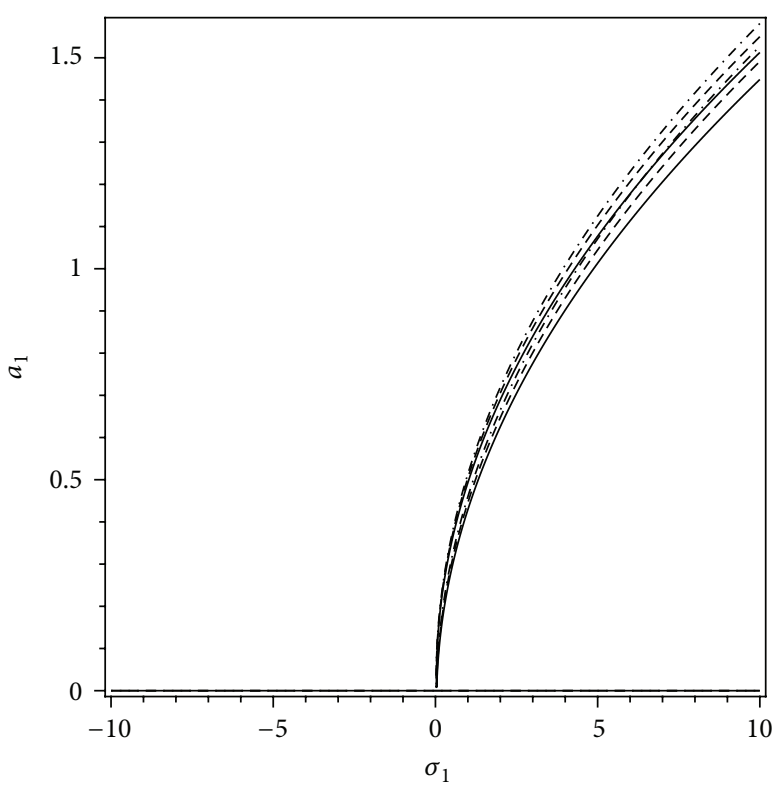

(a)

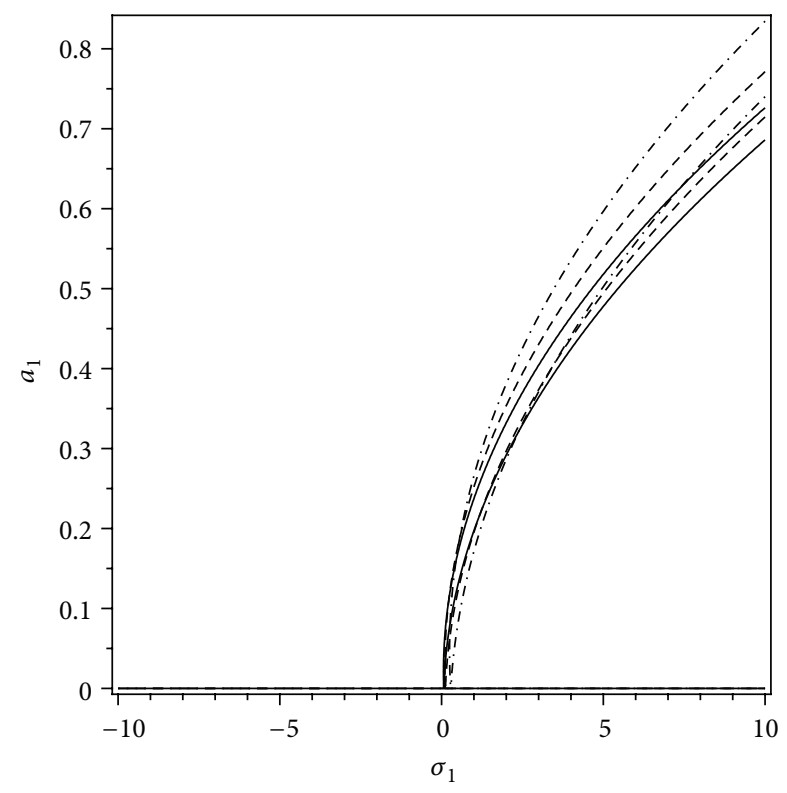

(b)

FIGURE 7: The hardening behavior of the first mode with subharmonic resonance. (a) The influences of the strut radius (solid line: $\bar{\rho}=0.02$; dash line: $\bar{\rho}=0.04$; dot-dash line: $\bar{\rho}=0.06$ ). (b) The influences of the core height (solid line: $h_{c} / h=0.74$; dash line: $h_{c} / h=0.82$; dot-dash line: $\left.h_{c} / h=0.9\right)$.

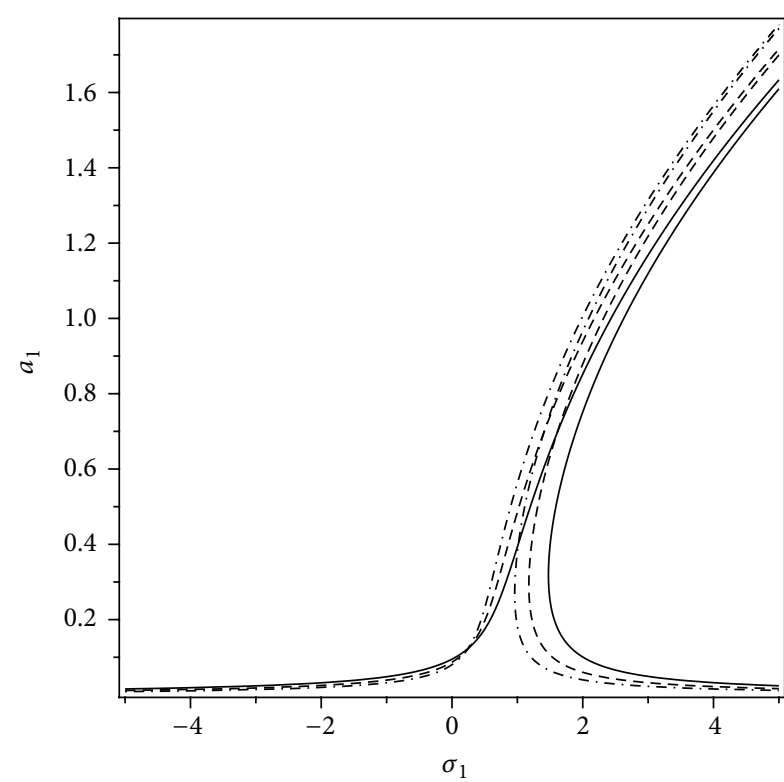

(a)

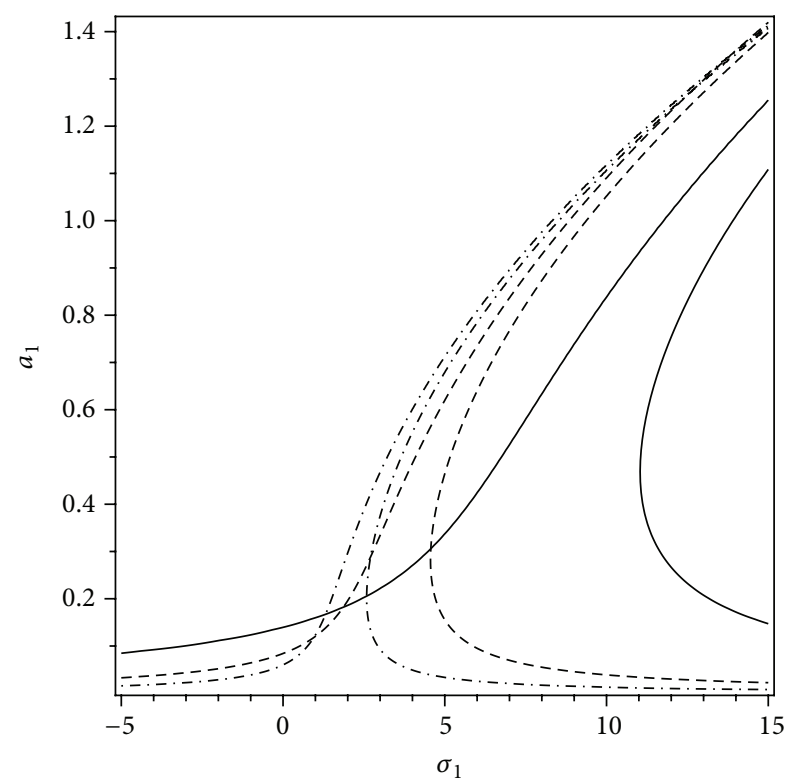

(b)

FIGURE 8: The hardening behavior of the first mode with superharmonic resonance. (a) The influences of the strut radius (solid line: $\bar{\rho}=0.02$; dash line: $\bar{\rho}=0.04$; dot-dash line: $\bar{\rho}=0.06$ ). (b) The influences of the core height (solid line: $h_{c} / h=0.74$; dash line: $h_{c} / h=0.82$; dot-dash line: $\left.h_{c} / h=0.9\right)$.

the structural parameters on the frequency parameter and the nonlinear term of the sandwich beam are studied in the paper for analyzing the variations of the hardening behavior.

The hardening nonlinearity bends the frequency response curve to the right. Generally, under the same excitation, the stronger the hardening-type nonlinearity, the smaller the response amplitude. We investigate the influences of strut radius and core height on the dynamical responses of the sandwich beam. Using the equations obtained in this paper, the influences of the other structural parameters of the sandwich beam on the vibrations can be conveniently analyzed. 
It is one of the effective methods to suppress the structure vibration by modifying the structural parameters, especially in the complex working circumstance. This method can avoid introducing new influence factors to the original system. Thus, it is very useful for the truss core sandwich structures which are usually used in aerospace vehicles. The free vibration and hardening behaviors under different resonances of the sandwich beam are studied in this paper. The parametrical study can provide the theoretical and data support for the design and vibration suppression of the truss core sandwich structures from the point of view of dynamics.

\section{Appendix}

The relations between the in-plane displacements and transverse displacement are

$$
\begin{aligned}
& u_{n}=S_{n} w_{n}^{2}, \\
& S_{n}=-\frac{n a_{1} \pi}{8} .
\end{aligned}
$$

The relations between the rotation angles and transverse displacement are

$$
\begin{aligned}
\phi_{n} & =K_{n} w_{n}, \\
K_{n} & =\frac{n b_{3} \pi-n^{3} b_{2} \pi^{3}}{n^{2} b_{1} \pi^{2}-1} .
\end{aligned}
$$

The coefficients in (20) are given as follows:

$$
\begin{aligned}
\gamma_{n} & =\frac{T_{2 n}}{T_{1 n}}, \\
\omega_{n}^{2} & =-\frac{T_{3 n}}{T_{1 n}}, \\
\alpha_{n} & =-\frac{T_{4 n}}{T_{1 n}}, \\
\beta_{n} & =\frac{T_{5 n}}{T_{1 n}}, \\
T_{1 n} & =\frac{c_{9}}{2}, \\
T_{2 n} & =\frac{c_{8}}{2}, \\
T_{3 n} & =\frac{n^{4} \pi^{4} c_{2}}{2}-\frac{n^{2} \pi^{2} c_{4}}{2}-\frac{n \pi K_{n} c_{3}}{2}+\frac{n^{3} \pi^{3} K_{n} c_{1}}{2}, \\
T_{4 n} & =-\frac{n^{4} \pi^{4}}{8}+\frac{n^{3} \pi^{3} S_{n} c_{6}}{2}-n^{3} \pi^{3} S_{n} c_{5}, \\
T_{5 n} & =\frac{c_{7}}{2},
\end{aligned}
$$

where

$$
\begin{aligned}
& a_{1}=\frac{h^{2}}{l^{2}}, \\
& b_{1}=-\frac{h^{2} \bar{h}_{c}^{2}}{2 l^{2}} \frac{\bar{A}_{1}}{\bar{C}} \text {, } \\
& b_{2}=\frac{h^{3} \bar{h}_{c}^{2}}{2 l^{3}} \frac{\bar{A}_{1}}{\bar{C}}+\frac{h^{3} \bar{h}_{c}}{l^{3}} \frac{\bar{B}_{1}}{\bar{C}}, \\
& b_{3}=-\frac{h}{l} \text {, } \\
& c_{1}=\frac{l}{3 h}\left(\frac{\bar{h}_{c}}{2}+\bar{h} \frac{\bar{B}_{1}}{\bar{A}_{1}}\right) \text {, } \\
& c_{2}=-\frac{1}{3}\left(\frac{\bar{h}_{c}^{2}}{2}+2 \bar{h} \frac{\bar{B}_{1}}{\bar{A}_{1}}+2 \frac{\bar{D}_{1}}{\bar{A}_{1}}\right), \\
& c_{3}=-\frac{l^{3}}{3 h^{3}} \frac{\bar{C}}{\bar{A}_{1}}, \\
& c_{4}=\frac{l^{2}}{3 h^{2}} \frac{\bar{C}}{\bar{A}_{1}} \text {, } \\
& c_{5}=\frac{2 l^{2}}{3 h^{2}}, \\
& c_{6}=\frac{2 l^{2}}{3 h^{2}}, \\
& c_{7}=\frac{l}{3 h \bar{A}_{1}} \text {, } \\
& c_{8}=\frac{l^{2}}{3 h^{2} \bar{A}_{1}} \text {, } \\
& c_{9}=\frac{l^{2}}{3 h^{2} \bar{A}_{1}}\left(2 \bar{I}_{01}+\bar{I}_{02}\right) \text {. }
\end{aligned}
$$

\section{Competing Interests}

The authors declare that they have no competing interests.

\section{Acknowledgments}

This research was supported by the National Natural Science Foundation of China (nos. 11402170 and 11402165).

\section{References}

[1] A. G. Evans, J. W. Hutchinson, N. A. Fleck, M. F. Ashby, and H. N. G. Wadley, "The topological design of multifunctional cellular metals," Progress in Materials Science, vol. 46, no. 3-4, pp. 309-327, 2001. 
[2] V. S. Deshpande and N. A. Fleck, "Collapse of truss core sandwich beams in 3-point bending," International Journal of Solids and Structures, vol. 38, no. 36-37, pp. 6275-6305, 2001.

[3] J. C. Wallach and L. J. Gibson, "Mechanical behavior of a threedimensional truss material," International Journal of Solids and Structures, vol. 38, no. 40-41, pp. 7181-7196, 2001.

[4] S. Hyun, A. M. Karlsson, S. Torquato, and A. Evans, "Simulated properties of Kagome and tehragonal truss core panels," International Journal of Solids and Structures, vol. 40, no. 25, pp. 6989-6998, 2003.

[5] T. Kim, H. P. Hodson, and T. J. Lu, "Fluid-flow and endwall heattransfer characteristics of an ultralight lattice-frame material," International Journal of Heat and Mass Transfer, vol. 47, no. 6-7, pp. 1129-1140, 2004.

[6] J.-H. Lim and K.-J. Kang, "Mechanical behavior of sandwich panels with tetrahedral and Kagome truss cores fabricated from wires," International Journal of Solids and Structures, vol. 43, no. 17, pp. 5228-5246, 2006.

[7] R. Biagi and H. Bart-Smith, "Imperfection sensitivity of pyramidal core sandwich structures," International Journal of Solids and Structures, vol. 44, no. 14-15, pp. 4690-4706, 2007.

[8] C.-H. Lim, I. Jeon, and K.-J. Kang, "A new type of sandwich panel with periodic cellular metal cores and its mechanical performances," Materials and Design, vol. 30, no. 8, pp. 30823093, 2009.

[9] J. S. Park, J. H. Joo, B. C. Lee, and K. J. Kang, "Mechanical behaviour of tube-woven Kagome truss cores under compression," International Journal of Mechanical Sciences, vol. 53, no. 1, pp. 65-73, 2011.

[10] J. Lou, L. Ma, and L.-Z. Wu, "Free vibration analysis of simply supported sandwich beams with lattice truss core," Materials Science and Engineering B: Solid-State Materials for Advanced Technology, vol. 177, no. 19, pp. 1712-1716, 2012.

[11] M. Xu and Z. Qiu, "Free vibration analysis and optimization of composite lattice truss core sandwich beams with interval parameters," Composite Structures, vol. 106, pp. 85-95, 2013.

[12] J. Lou, L. Z. Wu, L. Ma, J. Xiong, and B. Wang, "Effects of local damage on vibration characteristics of composite pyramidal truss core sandwich structure," Composites Part B: Engineering, vol. 62, pp. 73-87, 2014.

[13] F.-M. Li and X.-X. Lyu, "Active vibration control of lattice sandwich beams using the piezoelectric actuator/sensor pairs," Composites Part B: Engineering, vol. 67, pp. 571-578, 2014.

[14] Z.-G. Song and F.-M. Li, "Aeroelastic analysis and active flutter control of nonlinear lattice sandwich beams," Nonlinear Dynamics, vol. 76, no. 1, pp. 57-68, 2014.

[15] Y. Frostig and O. T. Thomsen, "High-order free vibration of sandwich panels with a flexible core," International Journal of Solids and Structures, vol. 41, no. 5-6, pp. 1697-1724, 2004.

[16] H. Biglari and A. A. Jafari, "High-order free vibrations of doubly-curved sandwich panels with flexible core based on a refined three-layered theory," Composite Structures, vol. 92, no. 11, pp. 2685-2694, 2010.

[17] J. E. Jam, B. Eftari, and S. H. Taghavian, "A new improved highorder theory for analysis of free vibration of sandwich panels," Polymer Composites, vol. 31, no. 12, pp. 2042-2048, 2010.

[18] E. Nilsson and A. C. Nilsson, "Prediction and measurement of some dynamic properties of sandwich structures with honeycomb and foam cores," Journal of Sound and Vibration, vol. 251, no. 3, pp. 409-430, 2002.
[19] Y. Q. Li and D. W. Zhu, "Free flexural vibration analysis of symmetric rectangular honeycomb panels using the improved Reddy's third-order plate theory," Composite Structures, vol. 88, no. 1, pp. 33-39, 2009.

[20] J. H. Zhang and W. Zhang, "Multi-pulse chaotic dynamics of non-autonomous nonlinear system for a honeycomb sandwich plate," Acta Mechanica, vol. 223, no. 5, pp. 1047-1066, 2012.

[21] X.-D. Yang, W. Zhang, and L.-Q. Chen, "Transverse vibrations and stability of axially traveling sandwich beam with soft core," Journal of Vibration and Acoustics, vol. 135, no. 5, Article ID 051013, 2013.

[22] R. Sahoo and B. N. Singh, "A new trigonometric zigzag theory for buckling and free vibration analysis of laminated composite and sandwich plates," Composite Structures, vol. 117, no. 1, pp. 316-332, 2014.

[23] R. K. Khare, T. Kant, and A. K. Garg, "Free vibration of composite and sandwich laminates with a higher-order facet shell element," Composite Structures, vol. 65, no. 3-4, pp. 405418, 2004.

[24] T. Sakiyama, H. Matsuda, and C. Morita, "Free vibration analysis of sandwich beam with elastic or viscoelastic core by applying the discrete green function," Journal of Sound and Vibration, vol. 191, no. 2, pp. 189-206, 1996.

[25] J. R. Banerjee, "Free vibration of sandwich beams using the dynamic stiffness method," Computers and Structures, vol. 81, no. 18-19, pp. 1915-1922, 2003.

[26] M. Ćetković and D. Vuksanović, "Bending, free vibrations and buckling of laminated composite and sandwich plates using a layerwise displacement model," Composite Structures, vol. 88, no. 2, pp. 219-227, 2009.

[27] A. J. M. Ferreira, "Analysis of composite plates using a layerwise theory and multiquadrics discretization," Mechanics of Advanced Materials and Structures, vol. 12, no. 2, pp. 99-112, 2005.

[28] H. G. Allen, Analysis and Design of Structural Sandwich Panels, Pergamon, Oxford, UK, 1969.

[29] A. H. Nayfeh and D. T. Mook, Nonlinear Oscillations, WileyInterscience, New York, NY, USA, 1979. 


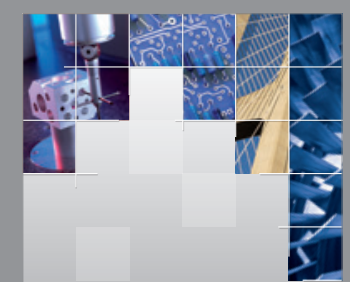

\section{Enfincering}
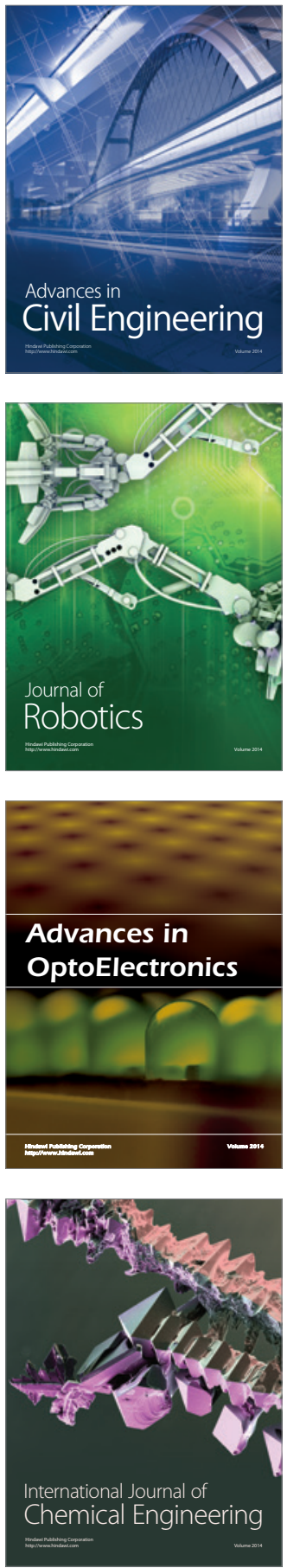

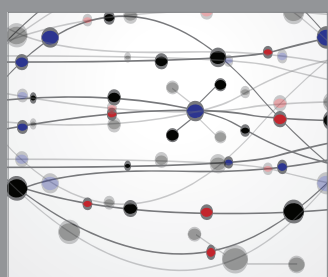

The Scientific World Journal

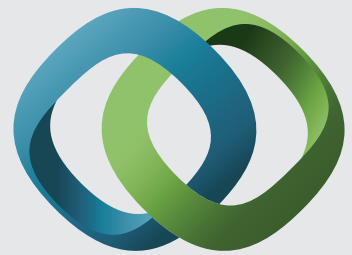

\section{Hindawi}

Submit your manuscripts at

http://www.hindawi.com
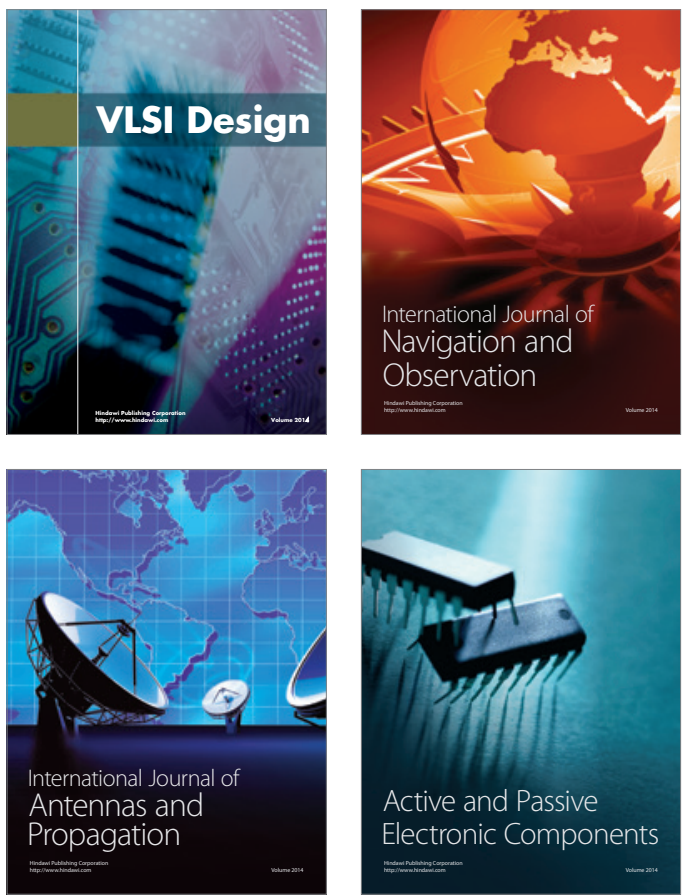
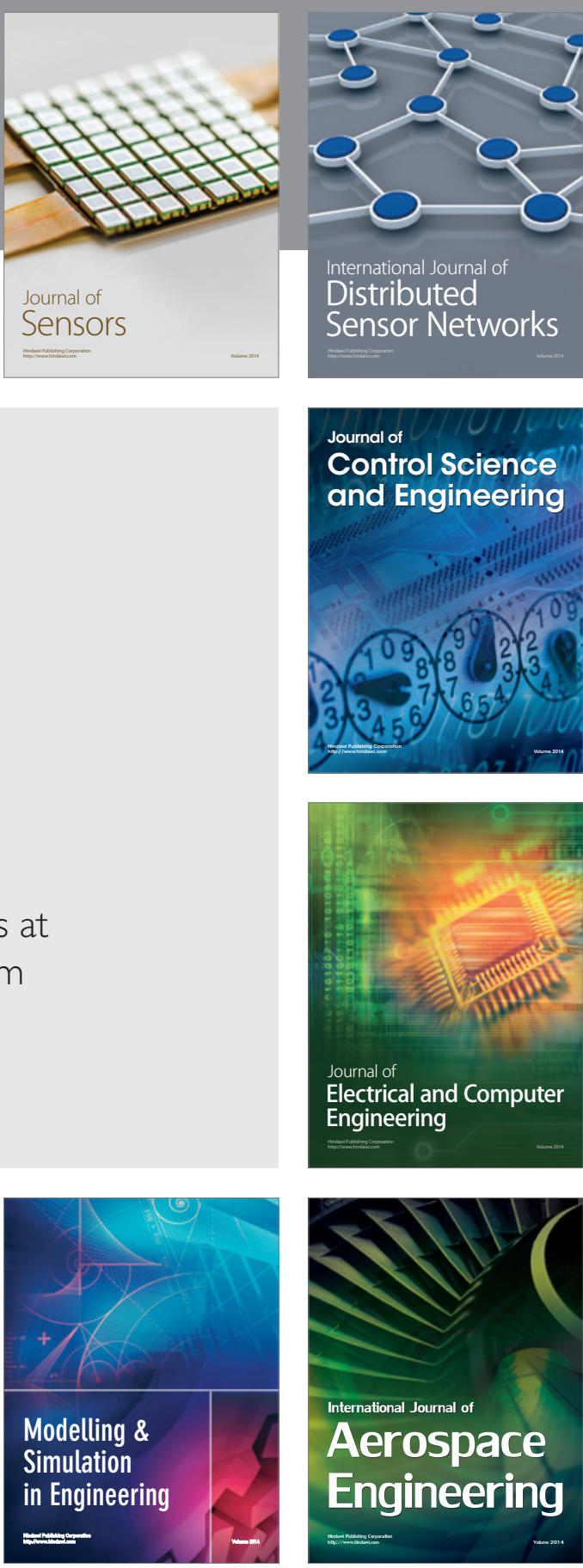

International Journal of

Distributed

Sensor Networks

Journal of

Control Science

and Engineering
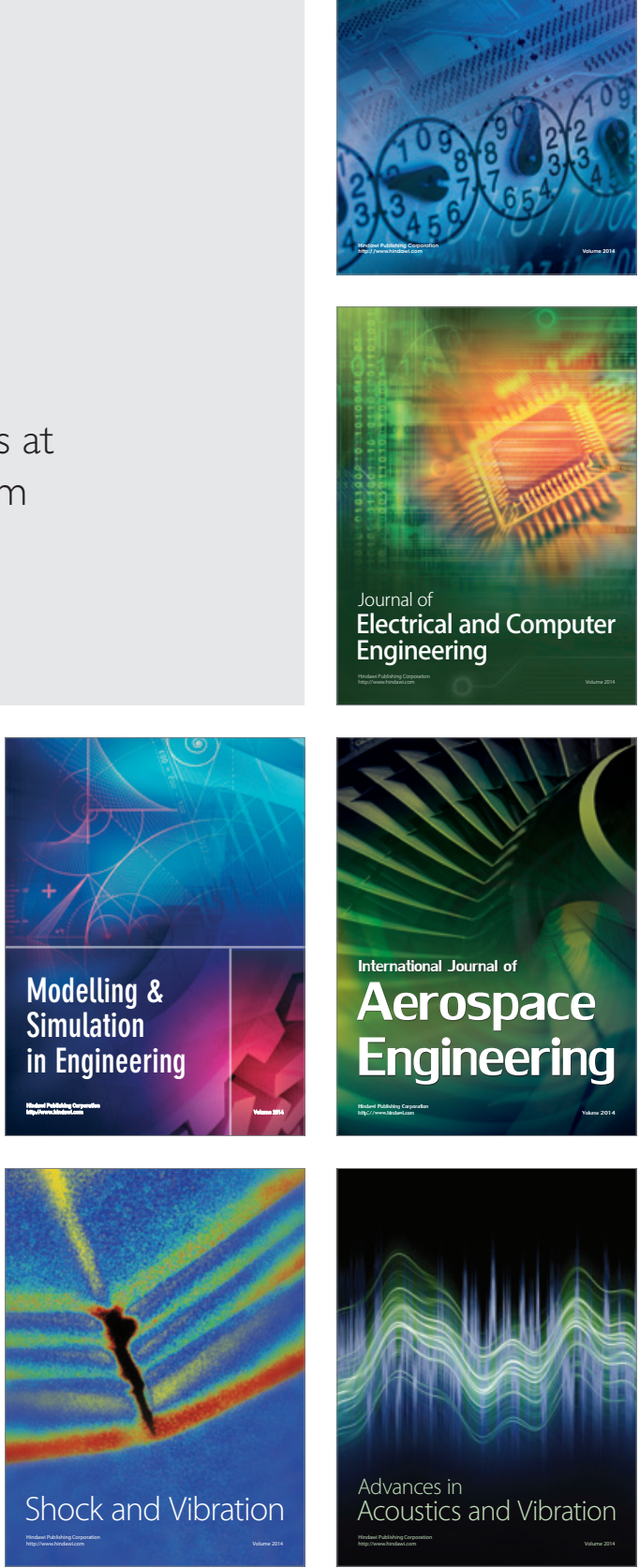